\title{
Fatigue failure results for multi-axial versus uniaxial stress screen vibration testing
}

\author{
Wayne E. Whiteman ${ }^{\mathrm{a}, *}$ and Morris S. Berman ${ }^{\mathrm{b}}$ \\ ${ }^{a}$ Department of Civil and Mechanical Engineering, US Military Academy, West Point, New York, USA \\ ${ }^{\mathrm{b}}$ Weapons \& Materials Research Directorate, US Army Research Laboratory, Adelphi, Maryland, USA
}

Received 12 November 2001

Revised 12 March 2002

\begin{abstract}
To date, the failure potential and prediction between simultaneous multi-axial versus sequentially applied uniaxial vibration stress screen testing has been the subject of great debate. In most applications, current vibration tests are done by sequentially applying uniaxial excitation to the test specimen along three orthogonal axes. The most common standards for testing military equipment are published in MIL-STD-810F and NAVMAT P-9492. Previous research had shown that uniaxial testing may be unrealistic and inadequate. This current research effort is a continuing effort to systematically investigate the differences between fatigue damage mechanisms and the effects of uniaxial versus tri-axial testing. This includes assessing the ability of the tri-axial method in predicting the formation of damage mechanisms, specifically looking at the effects of stress or fatigue failure. Multi-axial testing achieves the synergistic effect of exciting all modes simultaneously and induces a more realistic vibration stress loading condition. As such, it better approximates real-world operating conditions. This paper provides the latest results on the differences between multi-axial and uniaxial testing of a simple notched cantilever beam.
\end{abstract}

\section{Introduction and background}

The differences between simultaneous multi-axial versus sequentially applied uniaxial vibration stress screen testing in predicting failure potential has been the subject of great debate. Stress screen vibration testing is product-dependent and attempts to detect defective parts that might fail in a field environment, rather than simulate the characteristics of actual field conditions [1]. The purpose of stress screen vibration is to identify flaws that escape detection by other forms of testing. These flaws are often intermittent or latent potential defects in soldering, mounting, or wiring that appear only after certain thresholds of stress are crossed. The goal is to detect these problems during testing before a product goes out and experiences a failure in the real world [2].

*Corresponding author: Tel.: +1 845938 2956; Fax: +1 845938 5522; E-mail: Wayne-Whiteman@usma.edu.
To date, most stress screen vibration tests are conducted by sequentially applying uniaxial excitation to test articles along three orthogonal axes. In other words, the object is first vibrated up and down in the vertical axis. It is then removed from the fixture, rotated $90^{\circ}$, remounted, and tested in one horizontal direction. Finally it is removed, rotated, and tested along the third remaining axis. Both MIL-STD-810F, 1 Jan 2000, and NAVMAT P-9492, May 1979, provide guidance and specifications for the conduct of these tests. There are two major shortcomings of this sequential, uniaxial method. First, the time to mount, remount, set-up, and test articles multiple times can be excessive. A second, more important, shortcoming with this method is that the sensitive directions of many of the internal components of equipment being tested may not be aligned with the three orthogonal directions chosen for the test. The result is items that may pass uniaxial testing procedures but fail under operating conditions [2-5].

The US Army Research Laboratory at Adelphi, Maryland has a tri-axial vibration test system. This sys- 
tem utilizes specially developed hydrostatic bearings to achieve maximum drive stiffness in each of three orthogonal directions, with minimal cross-coupling between orthogonal directions. Using specialized mechanical constraints, the test platform can generate translational motions while all uncontrolled rotations are suppressed. This system was developed to address the shortcomings in uniaxial test methods and provide a test system that more closely approximates the life cycle environment of most Army materiel. The ultimate objective of the tri-axial system involves redefining vibration screening and testing procedures to properly validate the safety level of equipment, increase the efficiency of the test method, more effectively precipitate design and manufacturing flaws, and ensure the proper operation of critical components under battlefield operating conditions [3].

Some fatigue specialists maintain that the levels of stress caused by vibration are usually too low to contribute to fatigue damage, and that fatigue cracks start because of higher stresses present in the loading history. It is generally recognized, however, that large number of stress cycles generated by high-frequency vibration can substantially contribute to fatigue damage and in some circumstances cause failure without needing the occasional high load. Special analysis is often needed in these circumstances because the basic information about vibration is not in a form that can be used directly in a conventional Miner-type fatigue cumulative damage calculation. In spite of the fact that fatigue itself is not known to be very sensitive to frequency, tests using Power Spectral Density (PSD) to control the loading have shown a strong link between life and the PSD. Assuming a Guassian amplitude probability density distribution, the PSD fixes the peak and trough distribution of the vibration, thereby fixing the number and amplitude of motion reversals in a time-domain description of the stress history. This fact allows general fatigue life prediction from frequency-domain data [6]. The method of fatigue failure prediction in this current research effort takes advantage of these observations.

The test specimen in this paper is a simple notched cantilever beam structure. Tests are systematically conducted to experimentally determine observed differences in fatigue failure prediction. This paper provides uniaxial test results and some preliminary tri-axial test results. The uniaxial results indicate that there may be inadequacies in traditional uniaxial stress screen vibration test procedures. The tri-axial results further explore this hypothesis.

The paper begins with a brief description of the test set-up and procedures. Results and discussion follow for both uniaxial and tri-axial tests. The final section contains conclusions and recommendations for future work.

\section{Test set-up}

Both uniaxial and tri-axial random vibration excitation were applied to simple notched cantilever beam structures. The test specimens were manufactured from 2024-T4 Aluminum. A typical specimen is shown in Fig. 1. Dimensions are provided in Fig. 2. A Cartesian coordinate system is introduced to denote the direction of base excitation.

Aluminum is a nonferrous alloy and was chosen because it would not exhibit an endurance limit in the range of stresses experienced during the tests and would eventually fail due to fatigue. A notch was introduced around the entire circumference of the beams to facilitate the formation of the fatigue damage mechanism under repeated loads in a consistent manner.

Initial uniaxial tests were conducted on a Model PM75C-B, MB Dynamics Shaker. Later tests were conducted on the tri-axial vibration test system described earlier in this paper. The test specimens were mounted with simple plate fixtures. The uniaxial excitation was controlled by a Data Physics Corporation Controller. The multi-axial excitation was controlled by a custom-built system designed and specifically installed for use with the tri-axial shaker at the US Army Research Laboratory. Vibration output was acquired using an Ometron VPI Sensor laser interferometer. The test set-up for the initial uniaxial tests is depicted in Fig. 3. Figure 4 shows the tri-axial system used in later tests.

\section{Uniaxial test procedures and results}

To enable comparisons of test results, the same random vibration input spectrum was applied at specified input energy levels for each of the tests. Figure 5 shows the random vibration acceleration spectrum at the 4 gRMS input level applied during the uniaxial portion of the tests. The desired command spectrum is depicted.

Fatigue failure refers to the sudden, often catastrophic, separation of a specimen, or part, into two or more pieces after repeated loading. Failure takes place after initiation of a crack. The crack eventually becomes unstable and propagates to sudden breakage. Fatigue failure, for purposes of this study, was defined 


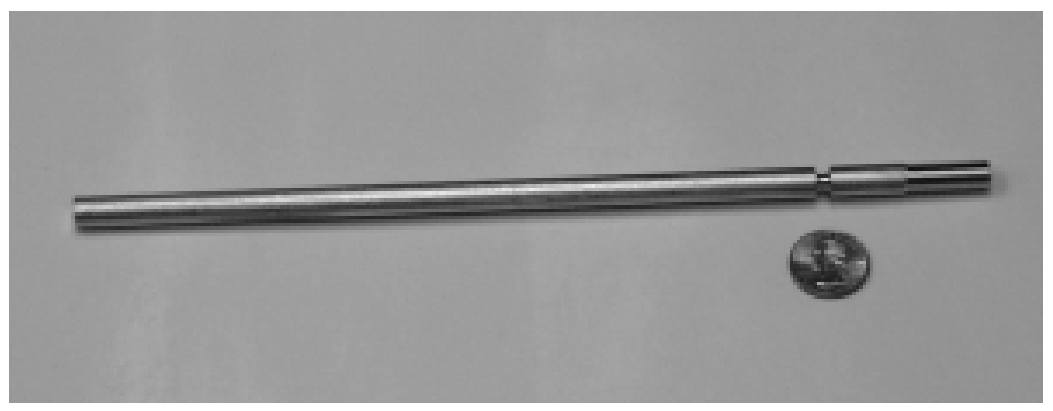

Fig. 1. Typical specimen.

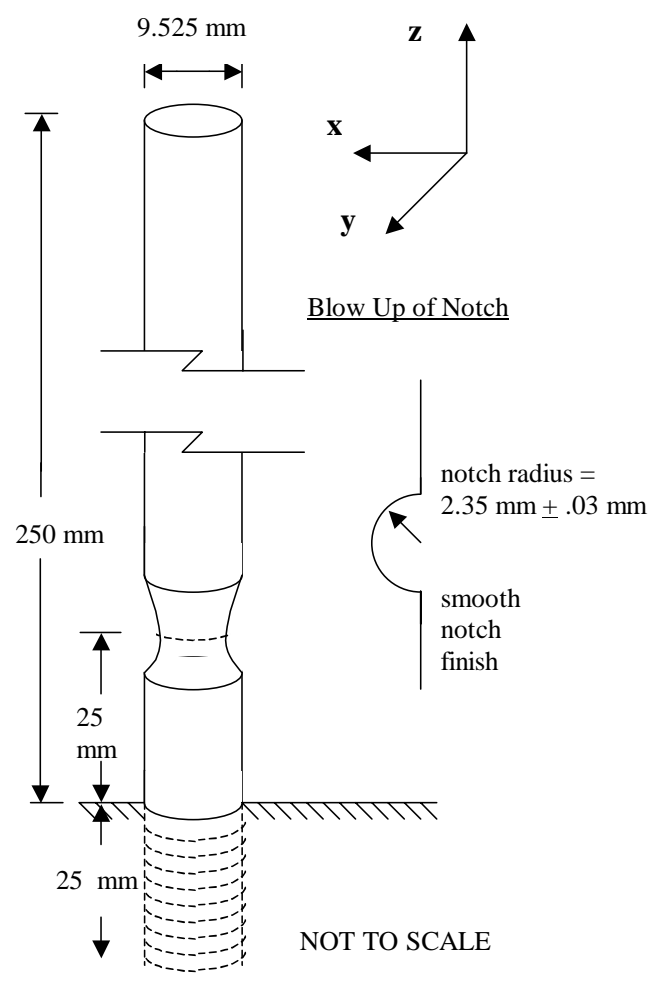

Fig. 2. Specimen dimensions.

to occur at the initiation of cracking. Crack initiation was detected by observing a drop in the first resonant frequency for the beam. While the specimen was subjected to the vibration input excitation described above, time history data of the beam's response was collected using the laser interferometer. A series of power spectral density (PSD) plots were calculated over the duration of the test. Each PSD spectrum was created from 5 frames of data sequentially averaged, each frame containing 500 samples collected at a sampling rate of $25.6 \mathrm{kHz}$ using Hanning windowing. These series of spectra were then arranged in a waterfall display showing the change in the beam's fundamental resonant fre- quency as a function of time. A typical waterfall plot from one of the tests is shown in Fig. 6.

The fundamental natural frequency for transverse vibration was observed by picking peaks from the PSD spectra. This first natural frequency was seen to shift dramatically as the specimen weakened from the onset of the tests through the formation of fatigue damage until final failure. At the beginning of the shaking sequence, the first resonant frequency was slightly above $80 \mathrm{~Hz}$. By the time complete fracture occurred, this fundamental resonant frequency had dropped below $50 \mathrm{~Hz}$. This observation motivated the decision to apply a random vibration input spectrum from 15 to $85 \mathrm{~Hz}$, versus a simple sinusoidal input at a particular frequency.

Peak values of each waterfall display were extracted to create scatter plots of frequency versus time. A trend of frequency shifting can be observed from these plots. Nonlinear regression techniques were used to curve fit this data. The characteristic equation for this curve fit was [7],

$$
\begin{gathered}
\left(\frac{x}{x_{\text {normalizationfactor }}}\right)^{\gamma} \\
+\left(\frac{y}{y_{\text {normalizationfactor }}}\right)^{\gamma}=1
\end{gathered}
$$

where $x$ represents the excitation frequency in hertz, and $y$ represents the time of the test in seconds.

A typical plot of curve fitted data of frequency versus time is shown in Fig. 7. Since failure was defined as occurring at the initiation of cracking, the fatigue failure time was calculated when the resonant frequency shifted from its original value by $5 \%$ as determined by the curve fit.

Initial tests were completed for transverse uniaxial vibration. Base excitation was identical in the $x$ or $y$ direction as shown in Fig. 2. For the first set of tests, specimens were excited until complete fatigue failure in 


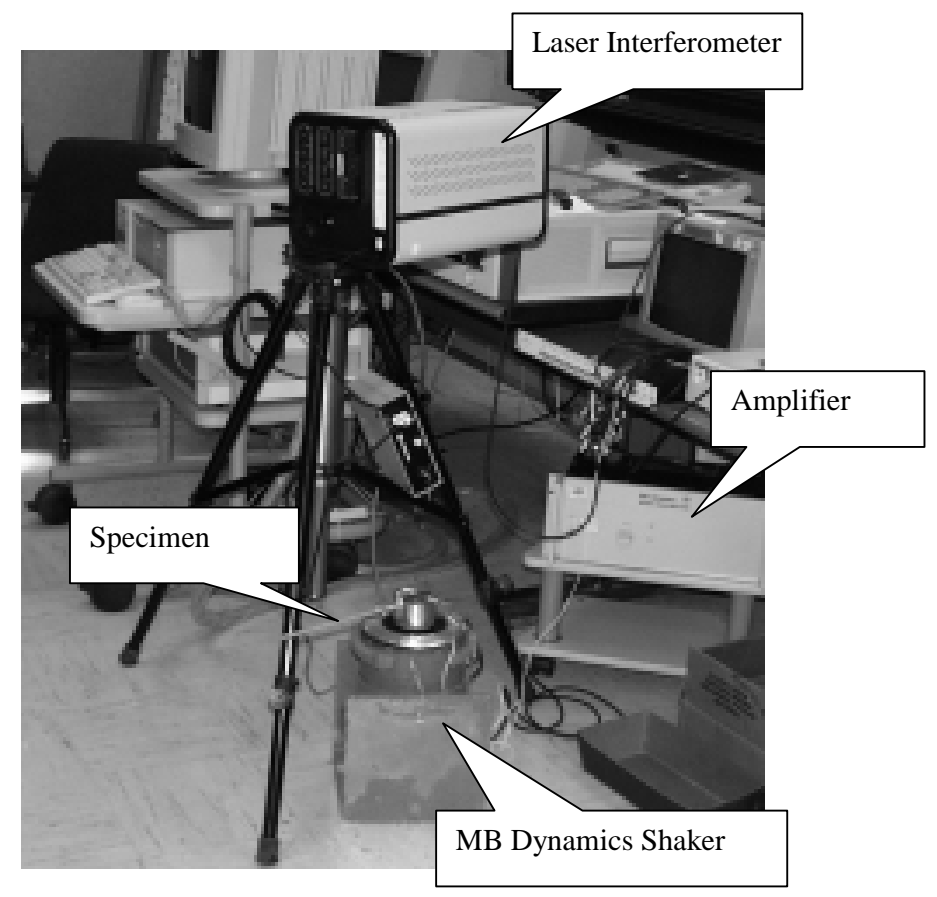

Fig. 3. Uniaxial test set-up.

the transverse direction. Results were plotted to show the input spectrum gRMS level on the ordinate axis and time to failure on the abscissa.

The resulting plots are analogous to typical fatigue strength life diagrams or S-N curves. An S-N curve plots stress levels versus number of cycles to failure in a typical fatigue test. If a test specimen is subjected to different levels of stress, fatigue cracks can develop and eventually lead to complete failure. As the test is repeated at higher stress levels, the number of cycles to failure becomes smaller. The results of these tests from a number of different stress levels are plotted as an S-N curve. The number of cycles to failure changes rapidly with stress level and may range over several orders of magnitude. For this reason, the cycle numbers are usually plotted on a logarithmic scale [8].

It has been proven that the stress response of a structure under dynamic loading is directly proportional to the velocity response [9-11]. Likewise, under random vibration, it can be shown that the stress spectrum is directly proportional to the velocity spectrum [12]. The acceleration spectrum, which is traditionally obtained and depicted using recorded accelerometer signals, can be related to the velocity spectrum. The time to failure under random excitation is directly proportional to the number of cycles to failure on S-N curves [6]. Accordingly, this time axis is plotted using a log scale to conform with typical S-N plots.
Figure 8 is a plot of 21 samples tested with uniaxial excitation in the transverse direction. Seven samples each were tested at the 3.5, 4.0, and 4.5 gRMS input levels. Since our sample sizes are small $(n=7)$, and we do not know the variance of the population, a t-sampling distribution with 6 degrees of freedom was used for statistical purposes. The $90 \%$ confidence intervals are shown on the graph and are given by:

$$
-t_{0.95} \leqslant \frac{(\bar{x}-\mu) \sqrt{n}}{\hat{S}} \leqslant t_{0.95}
$$

where

$$
\begin{aligned}
& \mu=\text { population mean } \\
& \bar{x}=\text { sample mean } \\
& \hat{S}=\text { sample standard deviation }
\end{aligned}
$$

The use of these confidence intervals is based on the assumption that the variance is the same at all gRMS levels. Although it appears that the spread of data is different at different gRMS levels, Bartlett's test on the data indicates that the variance is not statistically different at any reasonable choice for the level of significance. The data in Fig. 8 is consistent with expected simple fatigue tests for 2024-T4 Aluminum. The time to failure increases at lower input energy levels.

A similar procedure was repeated for the second set of tests except, in this series, the specimens were ex- 


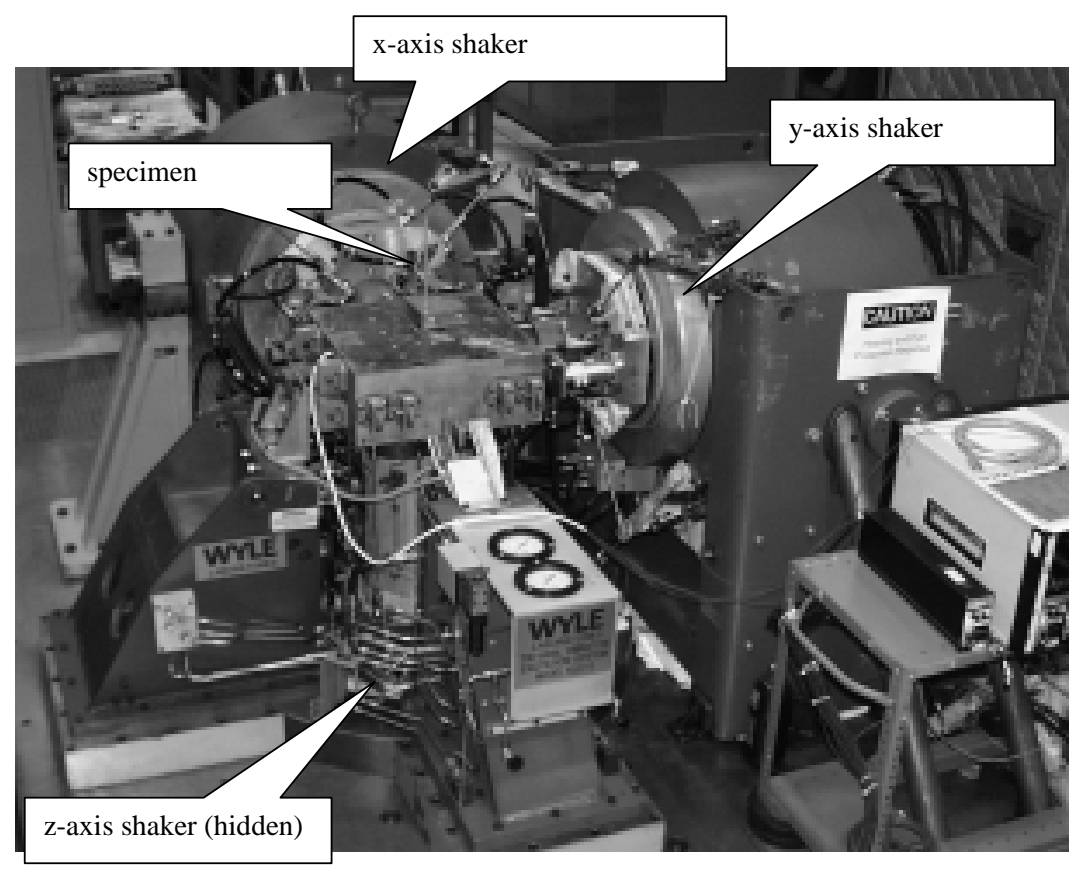

Fig. 4. Tri-axial test set-up.

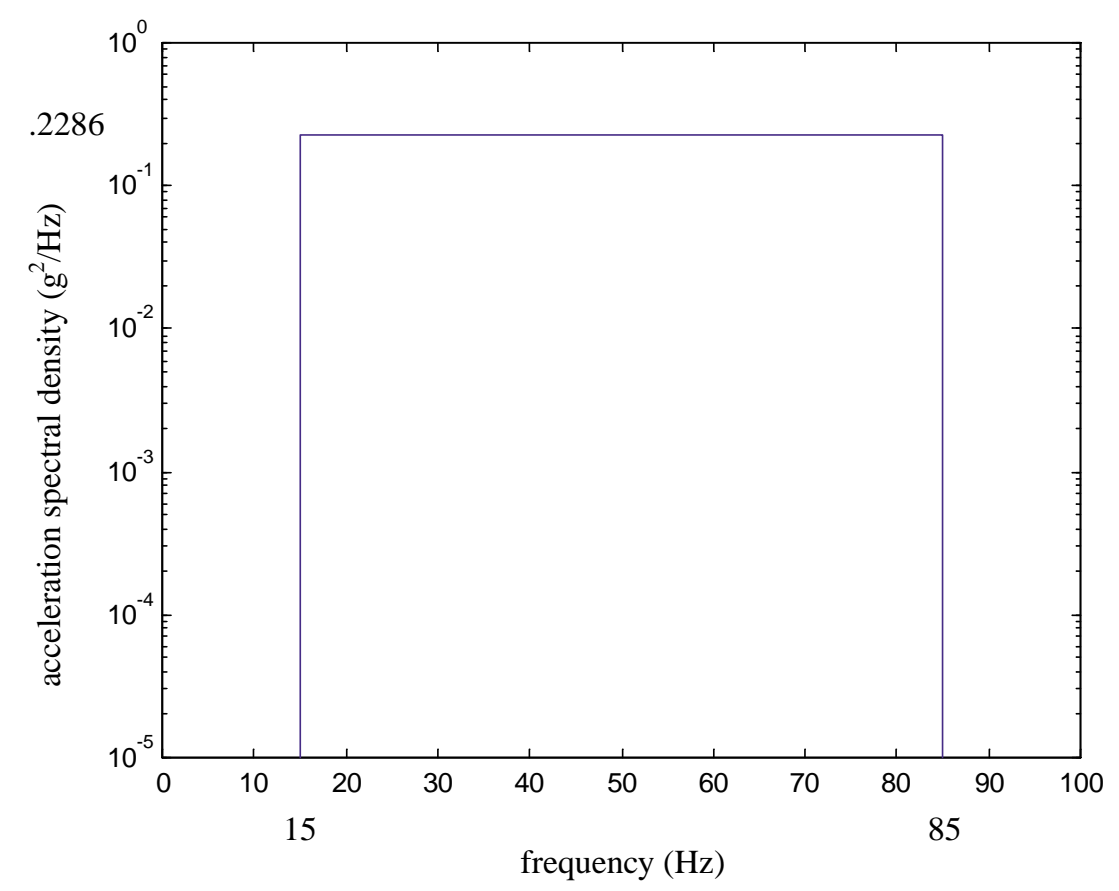

Fig. 5. Random vibration input acceleration spectrum-uniaxial shaker.

cited first in the axial, or $z$, direction for the previously determined time to failure followed by excitation in the transverse direction until fatigue failure. This allowed comparison to the previous uniaxial results for exci- tation in the transverse direction only. Once again, 7 samples were tested at the $3 \mathrm{gRMS}$ levels for a total of 21 samples.

Based on the results shown in Fig. 8, the initial ex- 


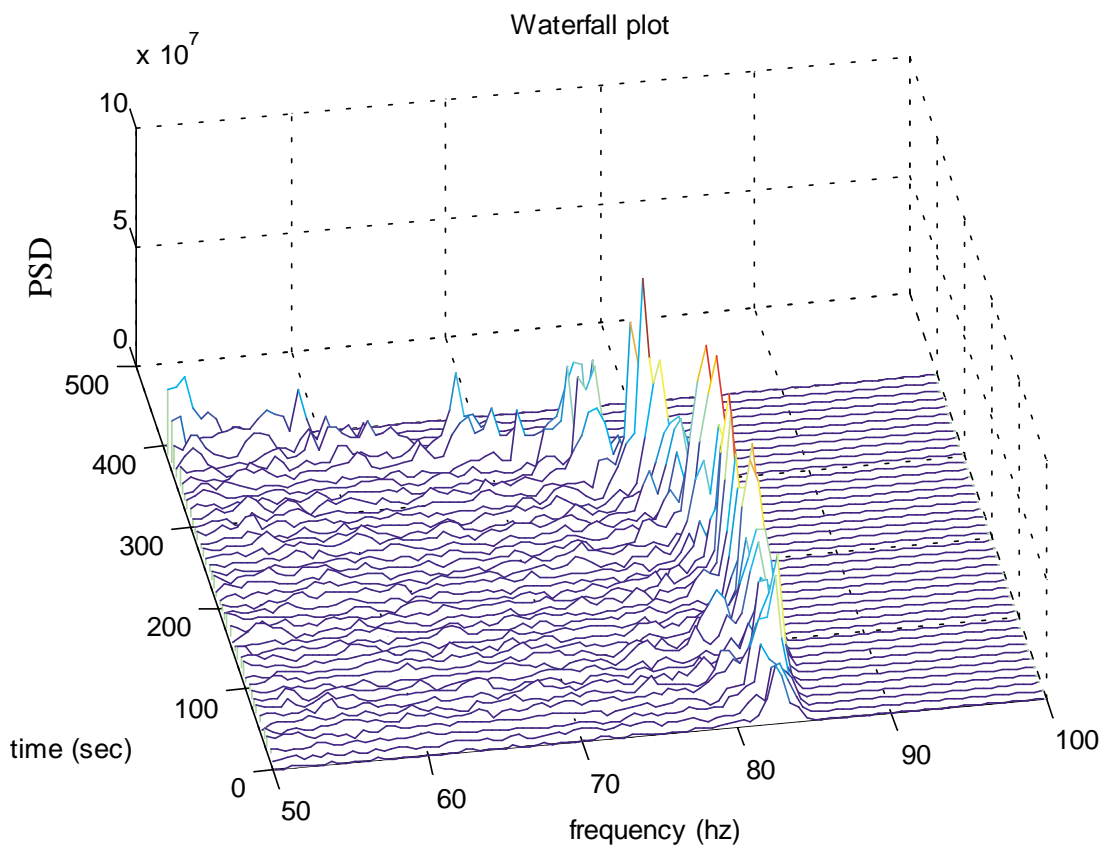

Fig. 6. Typicall waterfall display.

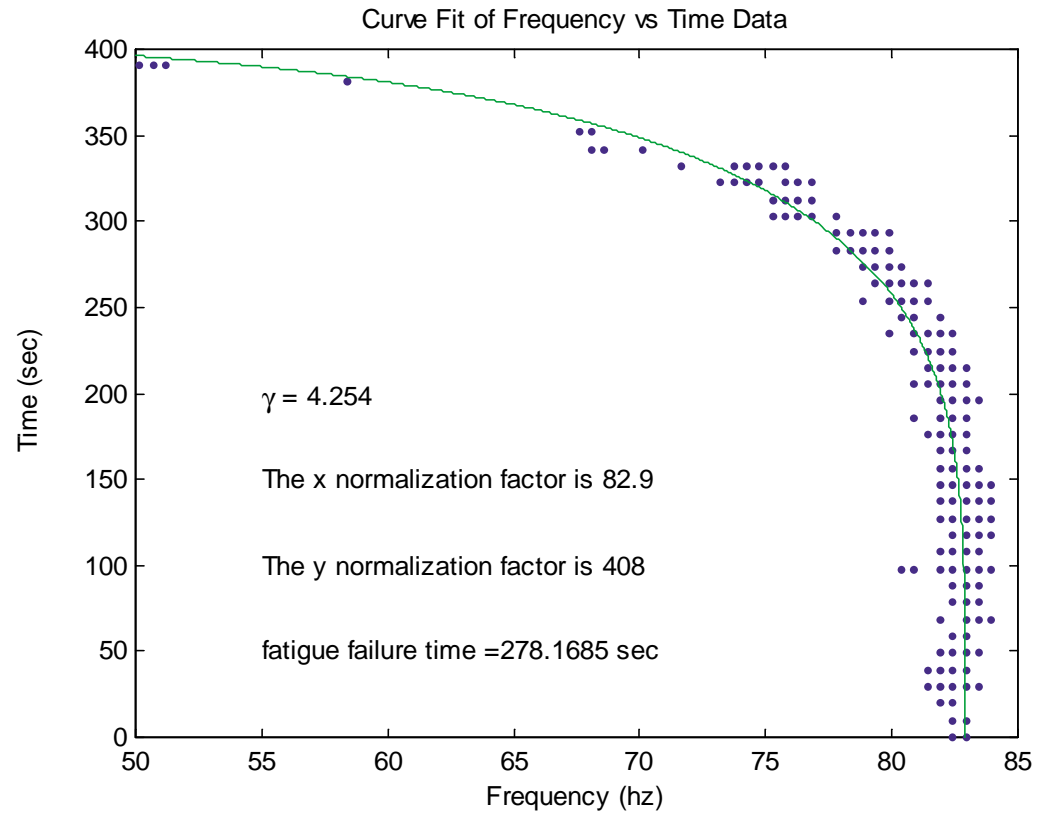

Fig. 7. Curve fit of frequency versus time data.

citation in the axial direction for this second series of tests was chosen to last 240 seconds at the $4.5 \mathrm{gRMS}$ level, 300 seconds at the 4.0 gRMS level, and $600 \mathrm{sec}-$ onds at the 3.5 gRMS level. These were the approximate average times to failure in the previous tests in the transverse direction only. As expected, the speci- mens did not fail since they were excited well below the natural frequency in the axial direction.

Now, using these same specimens, the tests were applied in the transverse direction until fatigue failure. Figure 9 shows the results for excitation in the axial direction followed by the transverse direction as com- 


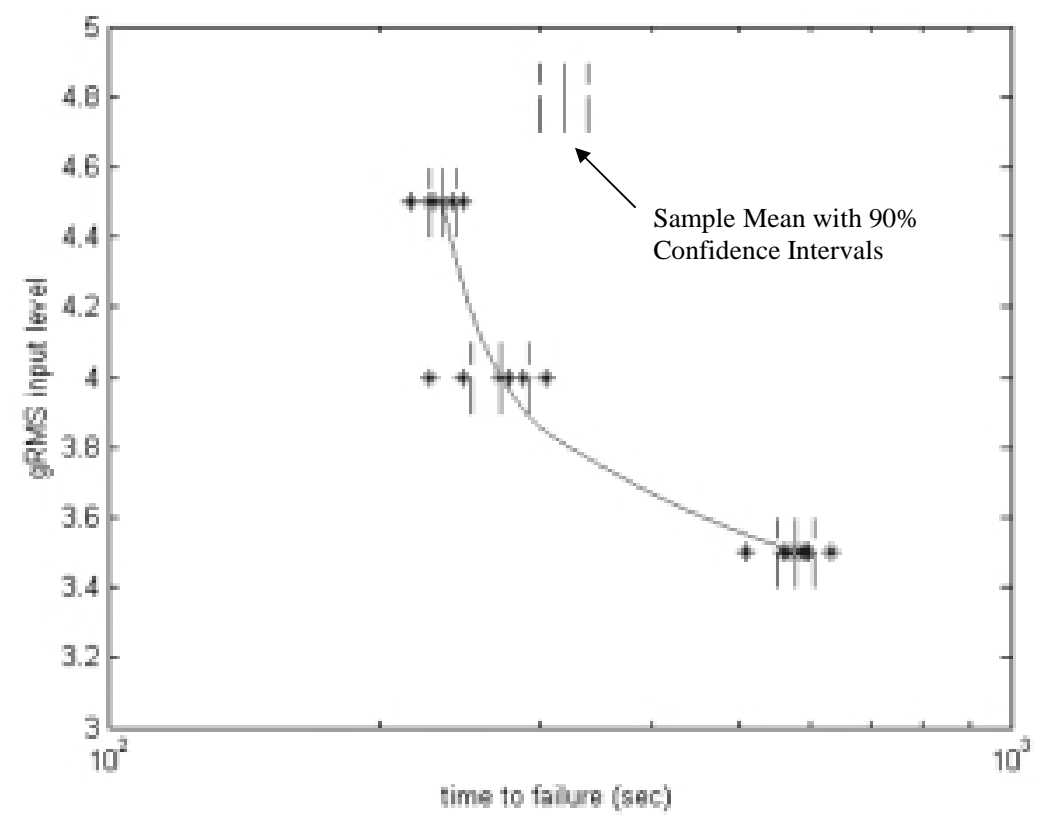

Fig. 8. Uniaxial results for excitation in the transverse direction only.

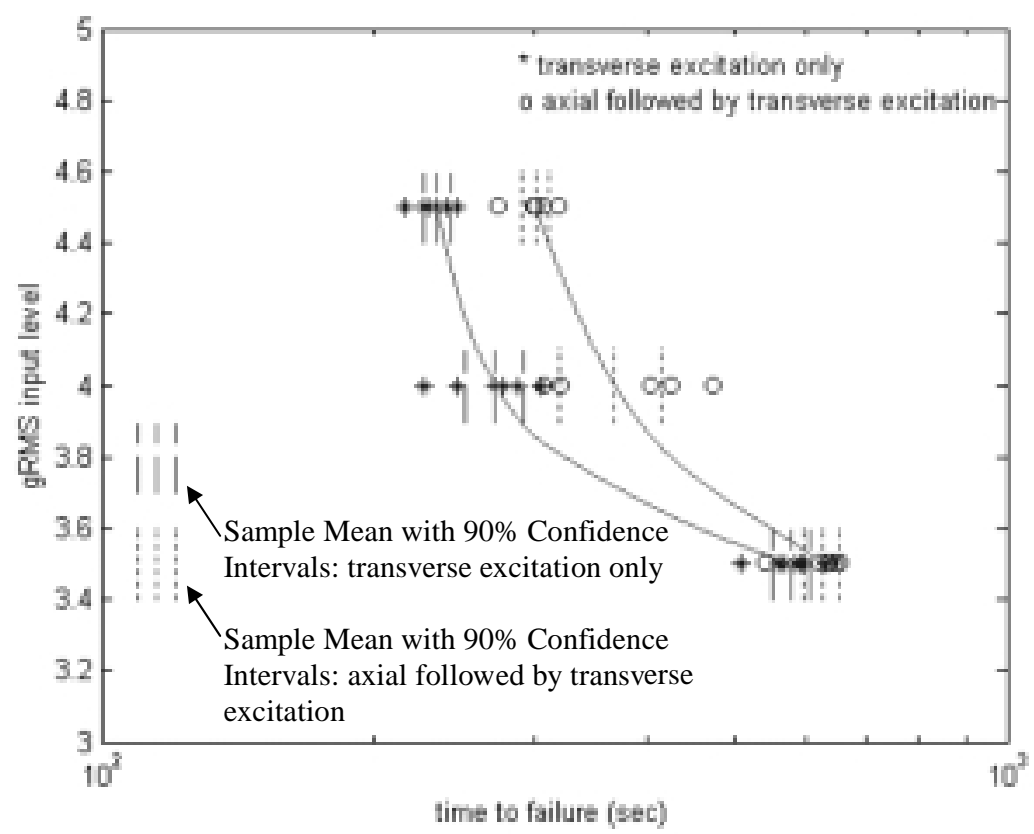

Fig. 9. Uniaxial results for axial followed by transverse versus transverse only.

pared to the previous series of tests for excitation to failure in the transverse direction only. Once again, for statistical purposes, the $90 \%$ confidence intervals are also shown on the graph.

These results were quite surprising and unexpected. It was expected that the preliminary excitation in the axial direction would weaken the specimens and cause more rapid failure when the transverse excitation was applied. Exactly the opposite occurred. From a macroscopic standpoint, it is believed that perhaps work or strain hardening took place during the axial excitation portion of the second set of tests and the specimens 


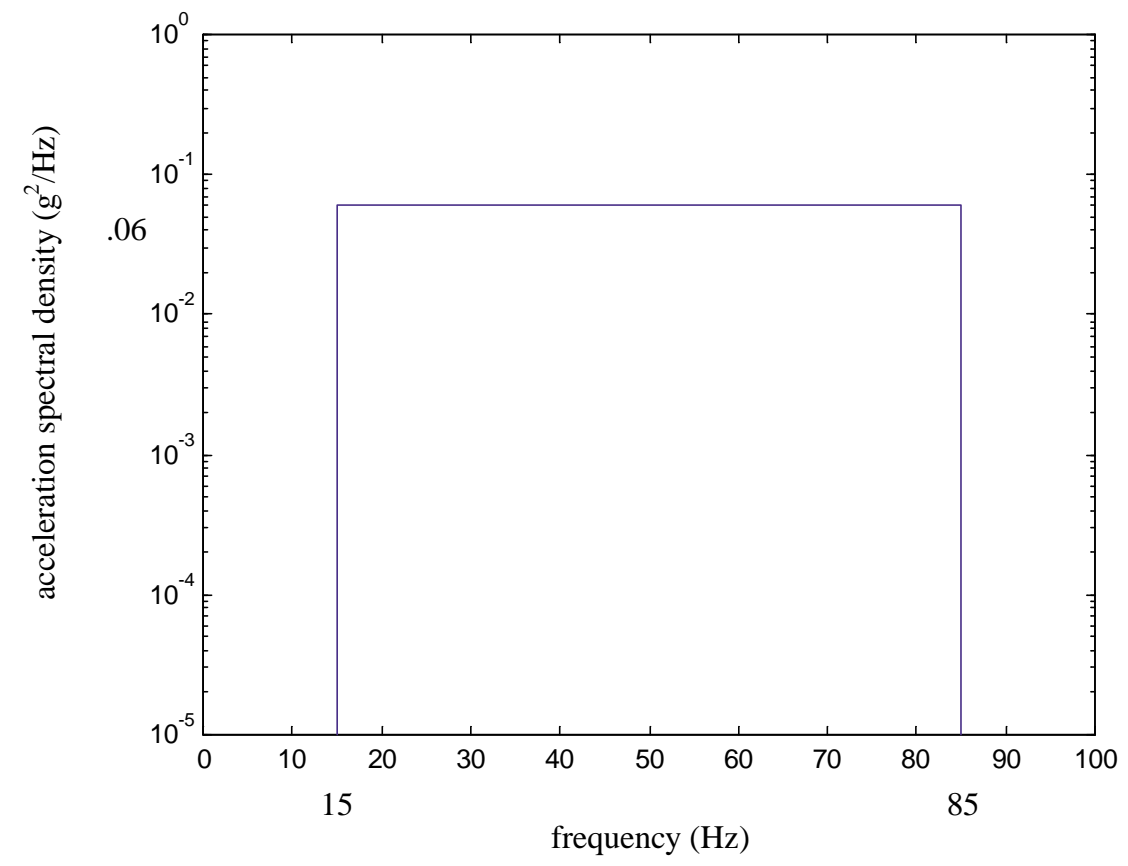

Fig. 10. Random vibration input acceleration spectrum-tri-axial shaker.

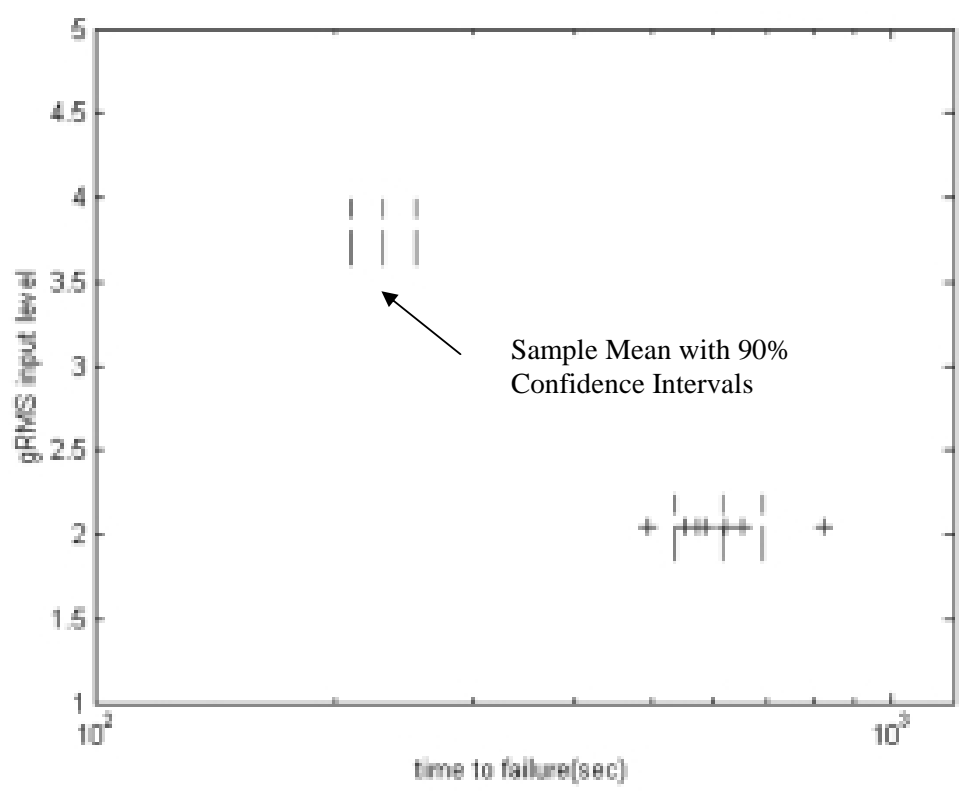

Fig. 11. Tri-axial results.

were actually more resistant to fatigue failure in the transverse direction as a result. These uniaxial results show an inadequacy of sequentially applied uniaxial test methods for this simple notch cantilever beam structure. The order in which the uniaxial excitation was applied during the test caused a variance in the results.

\section{Tri-axial test procedures and results}

Procedures for the tri-axial tests were very similar to the uniaxial tests. Once again, to enable the comparison 


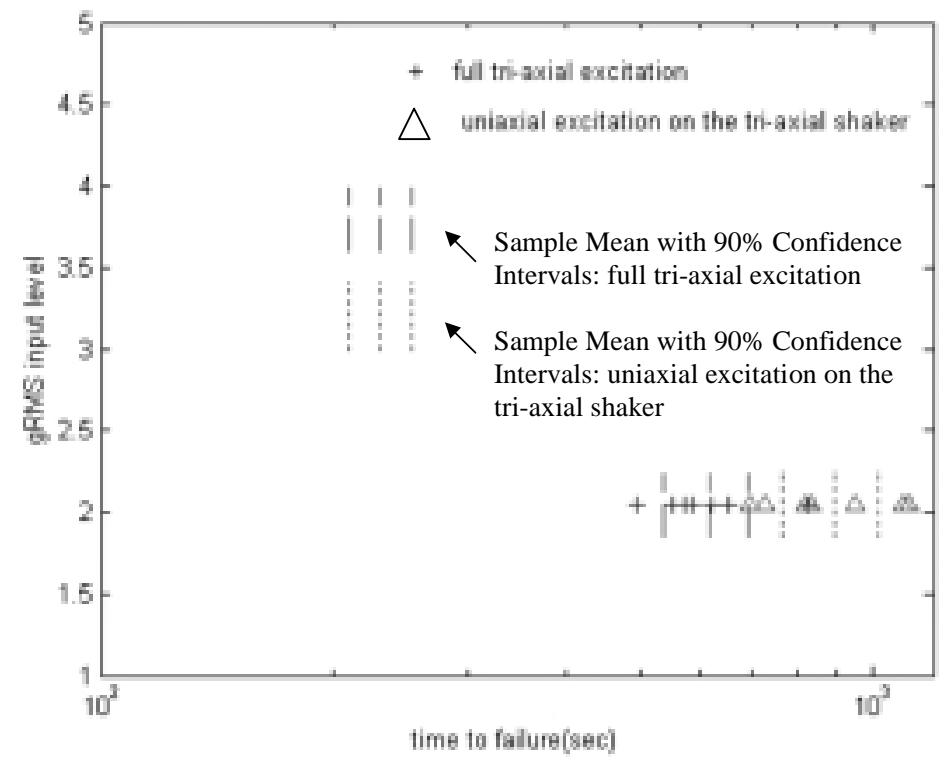

Fig. 12. Uniaxial results on the triaxis shaker versus full tri-axial tests.

of test results, the same random vibration input spectrum was applied at specified input energy levels for each of the tests. Figure 10 shows the random vibration input acceleration spectrum at the $2.05 \mathrm{gRMS}$ input level. The desired command spectrum is depicted.

Tests on the tri-axial shaker were completed both in the full tri-axial mode and were also completed uniaxially on the triaxis shaker for comparison. For the full tri-axial portion of the tests, the input acceleration spectrum depicted in Fig. 10 was applied simultaneously in the $x, y$, and $z$ directions. Figure 11 shows the results of these tests for 7 samples excited at the 2.05 gRMS level with the corresponding $90 \%$ confidence intervals.

For the uniaxial portion of the tests on the triaxis shaker, the input acceleration spectrum depicted in Fig. 10 was applied for the axis of interest. Using specially developed hydrostatic bearings to achieve maximum drive stiffness in this primary direction, minimal cross-coupling occurred in the other orthogonal directions.

During the uniaxial tests on the tri-axis shaker, the specimens were excited first in the axial, or $z$, direction for the previously determined time to failure from the full tri-axial tests, then in the $x$ direction for the previously determined time to failure from the full tri-axial tests, and finally in the y direction until fatigue failure. This allowed a means of comparison to the previous tri-axial results. Once again, 7 samples were tested.

It should be noted that, due to the geometry of the simple beam specimen, the stresses that ultimately caused fatigue failure were primarily in the $z$-direction. Any differences obtained in the results were due to the multi-axial excitation that simultaneously inputs energy in the off-axis directions and also due to different places of crack initiation. For example, an equal excitation on either the $x$ or on the $y$ axis produces the same stress level but on locals opposite 90 degrees meaning that when both excitations are carried out simultaneously the stress level is higher at a different local.

Based on the results shown in Fig. 11, the initial excitation in the $z$ and $x$ directions for this second series of tests was chosen to last 510 seconds. Then, using these same specimens, the tests were applied in the final orthogonal y direction until fatigue failure. Figure 12 shows these results as compared to the previous series of tri-axial tests alone, again with $90 \%$ confidence intervals indicated.

These results are again interesting and indicate inadequacies in uniaxial test methods. By comparison, the time to fatigue failure for the uniaxial tests were significantly longer than the tri-axial tests at the same input energy level. The result is that the structure could have passed the uniaxial testing procedure, but failed the tri-axial test at the same energy level.

Future uniaxial tests on the triaxis shaker are planned where 7 sets of specimens will be excited in the sequence of the $y$, then $z$, then $x$-axis direction and another 7 sets of specimens in the sequence of the $x$, then $y$, then $z$-axis direction. These tests will all be con- 
ducted at the $2.05 \mathrm{gRMS}$ level. These results should further confirm whether the order in which uniaxial excitation is applied causes variances in the results.

\section{Conclusions and recommendations}

The uniaxial results in this paper show an inadequacy of sequentially applied uniaxial test methods for the simple notch cantilever beam structure studied. The order in which the uniaxial excitation was applied during the test caused a variance in the results. It is reasonable to expect that the same variances occur with more complex items of Army hardware and equipment undergoing current uniaxial vibration stress screen test procedures.

The tri-axial tests also yielded interesting results that indicated inadequacies with uniaxial testing. First, the time to mount, remount, set-up, and test articles for the uniaxial tests versus the tri-axial tests were three times longer. More importantly however, was that the time calculated to fatigue failure for the uniaxial tests was significantly longer than the tests down at same input energy level in the tri-axial mode. These results show that this simple structure could have passed the uniaxial testing procedure, but failed a tri-axial test at the same energy level. Because simultaneous multi-axial excitation more closely approximates real-world operating condition by achieving the synergistic effect of exciting all modes simultaneously and induces a more realistic vibrational stress loading condition, it is expected that similar results would occur for more complex structures tested for real-world use. While these results do not definitively confirm all of the possible differences between uniaxial and multi-axial vibration environments, they are an important step in the systematic and rigorous investigation and yield interesting insights.

The experimental method described in this paper, of defining fatigue failure by observing resonant frequency shifts over time during stress screen vibration tests, can be easily applied to different geometries where multi-axial fatigue failure exists and to more complex and typical components in actual real-world systems. This will be the next step in the research plan. chanics Section of the Composite and Lightweight Structures Branch of the United States Army Research Laboratory. This support is greatly appreciated. The authors would also like to acknowledge the assistance of Major Douglas Matty from the Department of Mathematical Sciences at the United States Military Academy. Major Matty made a major contribution in the statistical analysis that occurred in the revision of this paper. The views expressed herein are those of the authors and do not purport to reflect the position of the US Military Academy, the Department of the Army, or the Department of Defense.

\section{References}

[1] K.Y. Chang and A.M. Frydman, Three-Dimensional Random Vibration Testing Definition and Simulation, Proceedings of the Institute of Environmental Sciences, 1990, pp. 129-139.

[2] H.P. Bausch, A. Franz and M. Lapin, Every Which Way But Loose, Sound and Vibration (November 1992), 6-11.

[3] M.T. Freeman, 3-axis Vibration Test System Simulates Real World, TEST Engineering and Management (December/January 1990-1991), 10-14.

[4] G.K. Hobbs, J.L. Holmes and R. Mercado, Stress Screening Using Multi-axial Vibration, SEECO 82, The Society of Environmental Engineers, London, England, 13-15 July 1982, pp. 47-59.

[5] G.K. Hobbs and R. Mercado, Six Degree of Freedom Vibration Stress Screening, The Journal of Environmental Sciences 29(6) (November-December 1984), 46-53.

[6] F. Sherratt, Vibration and Fatigue: Basic Life Estimation Methods, Journal of the Society of Environmental Engineers 22(4) (December 1983), 12-17.

[7] Characteristic equation for curve fit was developed through personal correspondence with Colonel Joseph Myers, Ph.D., Department of Mathematical Sciences, United States Military Academy, West Point, NY, July 2001.

[8] N.E. Dowling, Mechanical Behavior of Materials, Prentice Hall, New Jersey, 1993, pp. 343-346.

[9] F.V. Hunt, Stress and Strain Limits on the Attainable Velocity in Mechanical Vibration, J. Acoust. Soc. Amer. 32(9) (September 1960), 1123-1128.

[10] E.E. Ungar, Maximum Stresses in Beams and Plates Vibrating at Resonance, Trans. ASME, J. Engrg. Ind. 82B(1) (February 1962), 149-155

[11] S.H. Crandall, Relation between Strain and Velocity in Resonant Vibration, J. Acoust. Soc. Amer. 34(12) (December 1962), 1960-1961.

[12] H. Himelblau and M.J. Hine, Effects of Tri-axial and Uniaxial Random Excitation on the Vibration Response and Fatigue Damage of Typical Spacecraft Hardware, 66th Shock and Vibration Symposium, Arlington, VA, 1995.

\section{Acknowledgements}

This work was supported by the Experimental Me- 

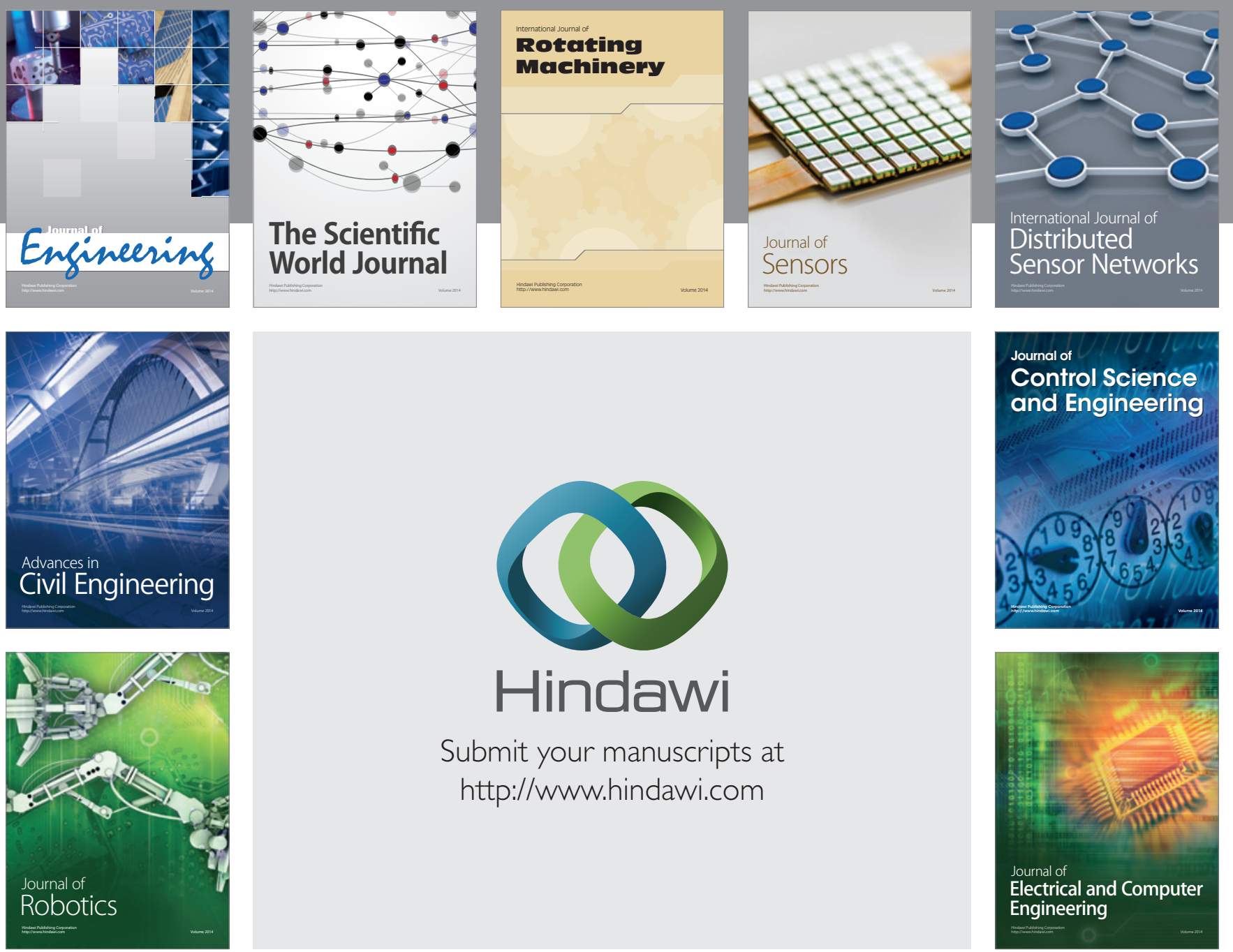

Submit your manuscripts at

http://www.hindawi.com
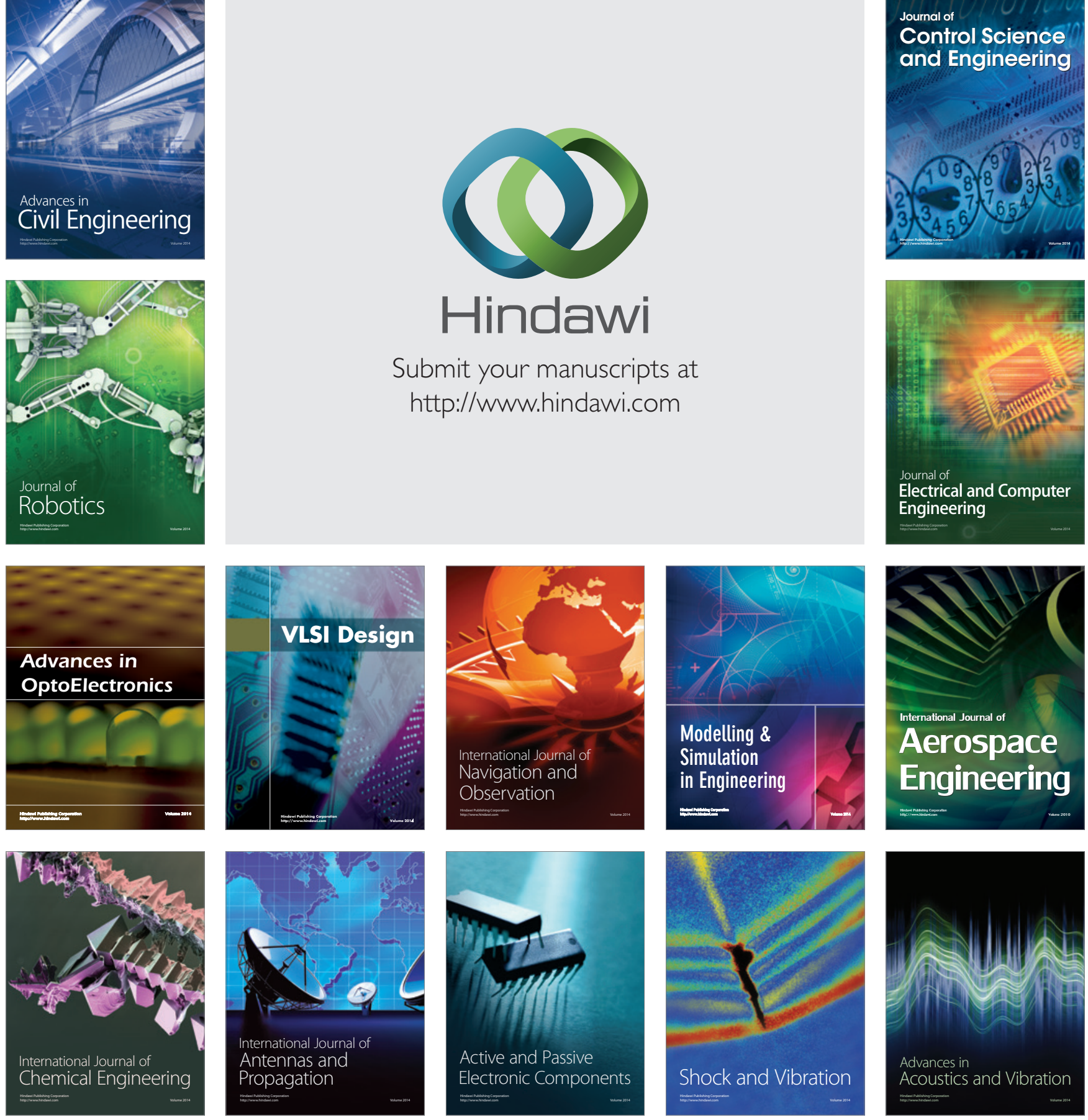